\title{
A functional polymorphism T309G in MDM2 gene promoter, intensified by Helicobacter pylori lipopolysaccharide, is associated with both an increased susceptibility and poor prognosis of gastric carcinoma in Chinese patients
}

Xiaolin Pan ${ }^{1 \dagger}$, Yuqin $\mathrm{Li}^{1+}$, Jin Feng ${ }^{2+}$, Xiaoyong Wang ${ }^{3^{*}}$, Bo Hao ${ }^{1}$, Ruihua Shi ${ }^{1}$ and Guoxin Zhang ${ }^{{ }^{*}}$

\begin{abstract}
Background: Studies on the association between MDM2 SNP309 (T > G) and gastric cancer have reported conflicting results. Thus, the aim of this study was to investigate whether MDM2 SNP309 is associated with susceptibility and prognosis of gastric carcinoma in Chinese patients.

Methods: Total of 574 gastric carcinoma cases and 574 age- and sex-matched healthy controls were included. MDM2 polymorphism was detected by PCR- RFLP and infection of Helicobacter pylori (H. pylori) by a validated serology test. The functionality of MDM2 SNP309, with or without $H$. pylori lipopolysaccharide (LPS), was examined by dual-luciferase assay. Kaplan-Meier survival curves were used to evaluate survival. Additional, a meta-analysis was conducted to verity the findings.

Results: MDM2 SNP309G/G genotype was associated with an increased risk of gastric carcinoma when compared with T/T genotype or T carriers (both $P<0.01$ ), and a joint effect between MDM2 SNP309G/G and H. pylori infection was observed to intensify gastric carcinoma risk. SNP309G/G was identified as an independent marker of poor overall survival of carcinoma. In vitro, the luciferase assay further showed an increased transcriptional activity of SNP309G allele compared with SNP309T allele, and the function of polymorphism T309G in MDM2 gene promoter was intensified by H. pylori LPS. Pooled results from the meta-analysis confirmed that SNP309G/G genotype had a significantly increased risk of gastric carcinoma compared with T/T genotype or T carriers, consistent with the casecontrol findings.

Conclusions: MDM2 SNP309G allele is associated with an increased risk and poor prognosis of gastric carcinoma in Chinese patients. Additional, there is a joint effect of MDM2 SNP309G/G allele and H. pylori infection on gastric carcinoma development, which may attribute to H. pylori LPS.
\end{abstract}

Keywords: Helicobacter pylori, Gastric carcinoma, MDM2, SNP, Lipopolysaccharide

\footnotetext{
* Correspondence: wxy20009@126.com; guoxinz@njmu.edu.cn

${ }^{\dagger}$ Equal contributors

${ }^{3}$ Department of Gastroenterology, Changzhou No.2 People's Hospital,

Affiliated with Nanjing Medical University, Changzhou 213000, China

1 Department of Gastroenterology, the First Affiliated Hospital of Nanjing

Medical University, Nanjing 210029, China

Full list of author information is available at the end of the article
} 


\section{Background}

The $p 53$ is well-known to be crucial in tumor prevention, which is activated by stress and oncogene activation as a transcription factor, and give rise to lots of cellular responses, such as cell cycle arrest, apoptosis, and senescence [1]. More than $50 \%$ of human carcinomas exist $p 53$ mutation or deletion [2]. MDM2 is an oncogene, encoding E3 ubiquitin ligase, which negatively regulates p53 transcriptional activity and protein stability [3], as it has been reported that the embryonic lethality of MDM2 null mice is caused by the uncontrolled activity of p53 and rescued by its deletion [4]. MDM2 overexpression could inhibit p53 function, and make damaged cells avoid the cell cycle checkpoint control and become carcinogenic $[5,6]$. In addition, there is a negative auto-regulatory feedback loop between MDM2 and p53, which plays an important role on regulating cell cycle progression, apoptosis and senescence [3].

A T-to-G single nucleotide polymorphism (SNP) is located 309 base pairs in promoter of $M D M 2$, which known as MDM2 SNP309 ( $\mathrm{T}>\mathrm{G}$ ), has been found to enhance the binding of the transcriptional activator specificity protein 1 (Sp1) to the P2 promoter of $M D M 2$, resulting in an increased MDM2 transcription and MDM2 protein expression, and a weaken of the p53 tumor suppression function [7]. It's important that the MDM2 SNP309G allele has been shown with an increased risk for the development of some tumors which express wild-type p53 [8-11].

H. pylori is a gram-negative pathogen that colonizes approximately $50 \%$ of the world's population, and is identified as a cause of gastric carcinoma development [12-14]. LPS of $H$. pylori is the constituent of its outer membrane, which has been reported to have the ability of promoting colonization of the mucus layer adjacent to the gastric epithelial surface [15], and enhancing proliferation and progression of gastric carcinoma [16,17].

Gastric carcinoma is the second most common reason for carcinoma-related deaths, accounting for more than 1 million deaths every year worldwide [18]. Several studies have investigated the association between MDM2 polymorphism and gastric carcinoma susceptibility [19-23]. However, these studies have produced inconsistent and inconclusive results. In our previous study, we reported that the MDM2 SNP309 (T > G) was associated with gastric carcinoma in Chinese patients, especially those with $H$. pylori infection [23]. However, that study was of small sample size and did not address any functional relation between MDM2 SNP309 ( $\mathrm{T}>\mathrm{G}$ ) and gastric carcinogenesis. Moreover, there has been no report on the association between MDM2 SNP309 ( $\mathrm{T}>\mathrm{G}$ ) and survival of gastric carcinoma patients in Chinese patients. Therefore, our aim is to investigate whether MDM2 SNP309 $(\mathrm{T}>\mathrm{G})$ is associated with susceptibility and prognosis to gastric carcinoma in Chinese patients.

\section{Methods}

\section{Study population}

574 gastric carcinoma patients and 574 healthy controls were included. All subjects reside in Jiangsu Province of China, and are unrelated ethnic Han Chinese. Patients were consecutively recruited from July 2005 to July 2009, at the First Affiliated Hospital of Nanjing Medical University. All cases were those newly histologically diagnosed with gastric carcinoma without previous chemotherapy or radiotherapy, which was staged and classified according to the American Joint Committee on Cancer Staging Manual, and Lauren's classification, when diagnosed $[24,25]$. All healthy controls were recruited from individuals living in the same residential areas who attended routine medical examination at the same hospital without no abnormal findings at the examination and were age- ( \pm 5 years $)$ and sex-matched to the cases. All subjects who had a history of tumors including gastric or other tumors or had undergone eradication treatment for $H$. pylori were excluded.

Venous blood $(2 \mathrm{ml})$ was collected from each subject at the entry of the study for the genotyping and detection of $H$. pylori infection. Gastric carcinoma patients were followed-up every 3 months by telephone or patient's reexamination to the hospital to update the progress of the disease.

This study has been approved by the Ethics Committee of the First Affiliated Hospital of Nanjing Medical University, and the written informed consent was obtained from all subjects, and the institutional ethics committees approving this research comply with acceptable international standards (the Treaty of Helsinki).

\section{Detection of $\mathrm{H}$. pylori infection}

The indirect solid phase immunochromatographic assay was applied to detect the infection of $H$. pylori according to its IgG antibodies (Genelabs Diagnostics Pty Ltd, Singapore), which has been validated in Chinese population with an accuracy of $92.3 \%$ [26].

\section{Genotype analysis}

Genomic DNA was extracted by proteinase $\mathrm{K}$ digestion and followed by phenol-chloroform extraction and ethanol precipitation from a leukocyte pellet. Polymerase chain reaction restriction fragment length polymorphism (PCRRFLP) was used to determine the genotype of MDM2 SNP309 [23]. In brief, the primer sequences were 5PCGCGGGAGTTCAGGGTAAAG-3P (forward) and 5PAGCTGGAGACAAGTCAGGACTTAAC-3P (reverse), which generated the 237-bp fragment. The PCR product was then digested by MSPA1I (New England Biolabs, Bevely, MA, USA). The wild-type (SNP309T) allele produces a single 237-bp fragment and the variant (SNP309G) allele produced two fragments of 189- and 
48 bp. Genotyping was performed without knowledge of the case or control status. The gel images were read independently by two research assistants. If a consensus was not reached on the tested genotypes, then the genotyping was repeated independently until a consensus was reached. In addition, to validate the RFLP method, 100 (50 from cases and 50 from controls) PCR products were selected randomly for direct sequencing with ABI 3700 sequencer, and the concurrence rate of these two methods was $99 \%$.

\section{Construction of reporter plasmids}

Because the association between MDM2 SNP309 ( $\mathrm{T}$ > G) and gastric carcinoma susceptibility, we then determined whether this polymorphism had an effect on gene expression in vitro. The MDM2 promoter-luciferase reporter plasmids containing either $551 \mathrm{~T}$ or $787 \mathrm{G}$ sequence were prepared by amplifying the 237 -bp MDM2 promoter region by using primers with restriction sites. The primers were 5P-GACGCTAGCTTTGTCG-CGCA GTTTCCACCG-3P (forward) and 5P-CGCGAAGCTT CTTCTTG-CTCCATCTTTCC-3P (reverse), including the NheI and HindIII restriction sites. We confirm the amplified fragments by sequencing. The amplified fragments and pGL3-basic vector (Promega, Beijing, China) were cleaved by using the NheI and HindIII enzymes (TaKaRa Biotech Co., Dalian, China). After the fragments were cloned into the pGL3-basic vector, the vectors were sequenced to confirm that there were no wrong.

\section{Cell culture}

Human gastric cancer cell lines AGS and MKN45 and mouse embryo fibroblast cell NIH-3 T3 (Institute of Cellular Research, Chinese Academy of Science, Shanghai, China) were maintained in RPMI-1640 medium supplemented with $10 \%$ fetal bovine serum (FBS). All the cells were incubated in a stable environment with $5 \% \mathrm{CO} 2$ at $37^{\circ} \mathrm{C}$ in a humidified incubator (Thermo, Forma Scientific, Inc., USA).

\section{Transient transfection and luciferase assays}

For transfection, NIH-3 T3, AGS and MKN45 cells were seeded onto 24-well plates (100,000 cells per well), and each well was cotransfected with $2.25 \mu \mathrm{g}$ of the vector DNA with either SNP309T or SNP309G allele and internal control $10 \mathrm{ng}$ pRL-SV40, in which a cDNA could encode the Renilla luciferase, and the pGL3-basic vector without any insert was applied as the negative control. After $24 \mathrm{~h}$ transfection, cells were lysed and luciferase activity was assayed by using the Dual-Luciferase Reporter Assay System (Promega) and normalized according to the Renilla luciferase activity. Each plasmid construct were done for independent triplicate.

\section{Purification and utilization of LPS}

LPS was extracted from $H$. pylori strains (26695) using the phenol-water extraction procedure previously described [27], and was quantitated using the purpald assay [28].

AGS cells were cotransfected with either MDM2 SNP309T or SNP309G luciferase reporters with Renilla luciferase reporter (as internal control) for $24 \mathrm{~h}$, followed by incubation with two concentrations $(0.1$ and $1 \mu \mathrm{g} / \mathrm{ml})$ of $H$. pylori LPS for $24 \mathrm{~h}$. Cells were lysed and luciferase activity performed by using the Dual-Luciferase Reporter Assay System (Promega) and normalized according to the Renilla luciferase activity. Each plasmid construct were done for independent triplicate.

\section{Statistical analysis}

Hardy-Weinberg equilibrium of alleles was evaluated by chi-square test. Comparison of age between cases and controls was evaluated by the Mann-Whitney $U$ test, and the difference in the distribution of genotypes between cases and controls was determined by chi-square test. The association between the MDM2 SNP309 and the risk for gastric carcinoma was estimated by odds ratios (OR) with the 95\% confidence intervals (CI). Logistic regression was used to control for selected potential confounders (sex, age, and H. pylori infection) and to assess crude and adjusted OR and 95\% CI. The difference in the levels of luciferase reporter gene expression between different constructs was examined by student's test. Kaplan-Meier was used to construct the cumulative overall survival curves and the difference was tested by the log-rank test, and Cox's proportional hazards model was used to perform the multivariate analysis to adjust for age, sex, TNM stage and $H$. pylori. All data analyses were done using SPSS software (version 11.0, Chicago, IL, USA). A $P$ value of $<0.05$ was considered statistically significant. Our sample size of 574 gastric carcinoma patients and 574 controls provided a $93 \%$ power to test an OR of 1.5 at the first error of 0.05 in a 2-sided test.

\section{Meta-analysis}

We identified related studies by searching the PubMed, Embase, Web of Science, China National Knowledge Infrastructure and Wanfang databases up to August 2012 with the search phrases of "murine double minute or MDM2" and "polymorphism(s) or SNP309" and "gastric or stomach" and "cancer or carcinoma or tumor or neoplasm". Additional studies were checked by screening reference lists of studies and reviews. The following criteria were used for literature selection: (a) the case-control study of the association between MDM2 SNP309 polymorphism and gastric carcinoma susceptibility; (b) genotypic frequencies in both cases and controls available. Only the study of most recent and complete was 
used in this meta-analysis. Hence, we included 5 studies in our meta-analysis, containing 1935 cases and 2953 controls. The following information was collected from each publication: the first author's name, year of publication, country, number of cases and controls, and MDM2 genotype information.

The odds ratios (ORs) with 95\% confidence intervals (CIs) were pooled to assess the association between MDM2 SNP309 and gastric carcinoma risk. The fixed effect model (Mantel-Haenszel method) and the random effect model (the Dersimonian and Laird method) were used to pool data from different studies [29,30]. If the heterogeneity between studies is absent, the fixed effect model was used; otherwise, the random effect model was applied. The $\mathrm{Z}$ test was used to determine the statistical significance of the OR. The chi-square-based $Q$ test and $\mathrm{I}^{2}$ were used to assess the statistical heterogeneity between studies, heterogeneity was considered statistically significant when $P \leq 0.05$ [30], and $\mathrm{I}^{2}$ was used to qualify variation in OR attributable to heterogeneity.
The publication bias was estimated by the Egger's test [31]. $P$ values $<0.05$ are considered statistically significant. All analysis was done by using the Review Manage (v.4.2) and Statistical Analysis System software (v.9.1.3, SAS Institute, Cary, NC).

\section{Results}

\section{Patient characteristics}

The cases and controls were well matched on sex and age. The median age was 58.56 years (range, $21-90$ years) for cases and 58.29 years (range, 22-88 years) for controls. There was no significance between cases and controls in sex distribution (Table 1). H. pylori infection was detected $408(71.1 \%)$ of cases and $390(67.9 \%)$ controls (Table 1).

\section{Association between MDM2 promoter SNP309 polymorphism and gastric carcinoma}

The genotype frequencies of the controls $(n=574)$ were consistent with the Hardy-Weinberg equilibrium distribution $(P=0.98)$.

\section{Table 1 Demographic, clinical and pathological characteristics of study subjects}

\begin{tabular}{|c|c|c|c|}
\hline Characteristics & Cases $(n=574)$ & Controls $(n=574)$ & $P$ value value \\
\hline Age (Mean $\pm S D$, years) & $58.56 \pm 12.09$ & $58.29 \pm 11.88$ & 0.71 \\
\hline Sex (Male/female) & $399 / 175$ & $399 / 175$ & 1.00 \\
\hline Seropositive rate of $H$. pylori infection & $71.1 \%$ & $67.9 \%$ & 0.25 \\
\hline \multicolumn{4}{|l|}{ Location of gastric carcinoma } \\
\hline Cardia & 181 & NA & \\
\hline Noncadia & 393 & NA & \\
\hline \multicolumn{4}{|l|}{ Vascular invasion } \\
\hline Absent & 363 & NA & \\
\hline Present & 211 & NA & \\
\hline \multicolumn{4}{|l|}{ Lymph node metastasis } \\
\hline Absent & 173 & NA & \\
\hline Present & 401 & NA & \\
\hline Liver metastasis & & NA & \\
\hline Absent & 512 & NA & \\
\hline Present & 62 & NA & \\
\hline \multicolumn{4}{|l|}{ Peritoneal dissemination } \\
\hline Absent & 503 & NA & \\
\hline Present & 71 & NA & \\
\hline \multicolumn{4}{|l|}{ TNM stage } \\
\hline IA & 96 & NA & \\
\hline $\mathrm{IB}$ & 79 & NA & \\
\hline$\|$ & 107 & NA & \\
\hline III & 173 & NA & \\
\hline IV & 119 & NA & \\
\hline Follow-up (months) & & NA & \\
\hline Median (Range) & $36(4-48)$ & NA & \\
\hline
\end{tabular}

Abbreviations: $S D$, standard deviation; NA, not applicable. 
Table 2 The genotype distributions of MDM2 SNP309 in the cases with gastric carcinoma and healthy controls

\begin{tabular}{|c|c|c|c|c|c|c|}
\hline & Controls $(n=574)$ & Patients $(n=574)$ & Crude OR $(95 \% \mathrm{Cl})$ & $P$ value & Adjusted OR $(95 \% \mathrm{Cl})^{\dagger}$ & $P$ value \\
\hline \multicolumn{7}{|l|}{ Genotype } \\
\hline $\mathrm{T} / \mathrm{T}$ & $34.7 \%$ & $30.1 \%$ & 1.00 & & & \\
\hline$T / G$ & $51.6 \%$ & $45.3 \%$ & $1.01(0.78-1.32)$ & 0.94 & $1.01(0.78-1.31)$ & 0.95 \\
\hline $\mathrm{G} / \mathrm{G}$ & $13.7 \%$ & $24.6 \%$ & $2.05(1.46-2.89)$ & $<0.01$ & $2.06(1.46-2.90)$ & $<0.01$ \\
\hline \multicolumn{7}{|c|}{ Recessive model } \\
\hline $\mathrm{T}$ carriers & $86.3 \%$ & $75.4 \%$ & 1.00 & & & \\
\hline $\mathrm{G} / \mathrm{G}$ & $13.7 \%$ & $24.6 \%$ & $2.04(1.51-2.77)$ & $<0.01$ & $2.05(1.51-2.78)$ & $<0.01$ \\
\hline \multicolumn{7}{|c|}{ Dominant model } \\
\hline $\mathrm{T} / \mathrm{T}$ & $31.2 \%$ & $30.1 \%$ & 1.00 & & & \\
\hline G carriers & $68.8 \%$ & $69.9 \%$ & $1.23(0.96-1.58)$ & 0.10 & $1.23(0.96-1.58)$ & 0.10 \\
\hline
\end{tabular}

${ }^{\dagger}$ Adjusted for age, sex, and $\mathrm{H}$. pylori infection.

Abbreviations: $\mathrm{OR}$, odds ratio; $\mathrm{Cl}$, confidence interval.

The genotype distributions in $M D M 2$ polymorphism of cases and controls are shown in Table 2. The MDM2 SNP309 genotype frequencies were 30.1\% (T/T), 45. 3\% $(\mathrm{T} / \mathrm{G})$ and $24.6 \%(\mathrm{G} / \mathrm{G})$ in cases, and $34.7 \%(\mathrm{~T} / \mathrm{T}), 51.6 \%$ $(\mathrm{T} / \mathrm{G})$ and $13.7 \%(\mathrm{G} / \mathrm{G})$ in control subjects $(P<0.01)$. The SNP309G/G genotype, but not SNP309T/G genotype, was associated with an increased risk of gastric carcinoma; the adjusted OR (95\%CI) was 2.06 (1.46-2.90) and $1.01(0.78-1.31)$ for SNP309G/G homozygotes and SNP309T/G heterozygotes, respectively, compared with the SNP309T/T homozygotes. In the recessive model, the SNP309G/G homozygote was associated with a 2.05fold increased risk of gastric carcinoma, compared with the T carriers (adjusted OR, 2.05; 95\% CI, 1.51-2.78; $P<$ 0.01 ). Table 3 shows a significantly increased risk of gastric carcinoma in patients with SNP309G/G genotype and $H$. pylori infections (adjusted OR, 2.44, which was greater than $1.14 \times 1.94=2.21$ ), compared with $\mathrm{T}$ carriers without $H$. pylori infection.

\section{Association of MDM2 SNP309 and gastric carcinoma in} relation to the location, metastatic status and TNM stage of gastric carcinoma

The risks of subtypes of gastric carcinoma for SNP309G/ $\mathrm{G}$ were significantly increased when compared with T carriers, especially non-cardiac carcinoma (adjusted OR, 2.18; 95\% CI, 1.57-3.04), lymph node metastasis present carcinoma (adjusted OR, 2.25; 95\% CI, 1.63-3.12), liver metastasis present carcinoma (adjusted OR, 3.34; 95\% CI, 1.84-6.08), peritoneal dissemination present carcinoma (adjusted OR, 2.13; 95\% CI, 1.18-3.84), and advanced stages carcinoma (adjusted OR, 2.30; 95\% CI, 1.52-3.49 for stage III, and 2.29; 95\% CI, 1.42-3.69 for stage IV; Table 4).

\section{Kaplan-Meier survival cures}

Overall, 133 patients with gastric carcinoma were followed up for a median (range) of 36 (4-48) months. Of the 101 patients with TNM stages II (T1N2-N3M0, T2N1N2M0, T3N0-N1M0, or T4aN0M0) to IV (TanyNanyM1), SNP309 (G/G) homozygotes had a significant association of poor overall survival $(P<0.01$, Figure $1 \mathrm{~B})$. The difference in survival among SNP309 genotypes in 32 stage I (T1N0-N1M0 or T2N0M0) patients was not significant $(P=0.50$, Figure $1 \mathrm{~A})$. In addition, Cox proportional hazards analysis showed that SNP309 (G/G) was an independent factor for poor prognosis of gastric carcinoma with TNM II -IV stages (hazard ratio, 2.13; 95\% CI, 1.04 to $4.36 ; P=0.04$ ) to adjust for sex, age, TNM stage and H. pylori infection.

\section{Effects of MDM2 SNP309 (T > G) on transcriptional activity of MDM2 gene}

As shown in Figure 2, the vectors with SNP309G allele produced a $100 \%$ to $300 \%$ increase in the relative

Table 3 Association between MDM2 SNP309 and gastric carcinoma in relation to $H$. pylori infection

\begin{tabular}{llll}
\hline MDM2 & H. pylori infection & Odds ratio ${ }^{\dagger}$ (95\% Confidence interval) & $\boldsymbol{P}^{\text {value }}$ \\
\hline T carriers & Negative & 1.00 & 0.37 \\
T carriers & Positive & $1.14(0.86-1.51)$ & 0.02 \\
GG & Negative & $1.94(1.11-3.38)$ & $<0.01$ \\
GG & Positive & $2.44(1.62-3.66)$ & \\
\hline
\end{tabular}

${ }^{\dagger}$ Adjusted for age and sex. 
Table 4 Association between MDM2 SNP309 and gastric carcinoma in relation to location, metastasis and TNM stage

\begin{tabular}{|c|c|c|c|c|c|}
\hline \multirow[t]{2}{*}{ Variable } & \multicolumn{2}{|c|}{ MDM2 SNP309 genotype (Control/Case) } & \multicolumn{2}{|c|}{ Adjusted OR $(95 \% \mathrm{Cl})^{\dagger}$} & \multirow[t]{2}{*}{$P$ value } \\
\hline & $\mathrm{TT}+\mathrm{TG}$ & GG & $\mathrm{TT}+\mathrm{TG}$ & GG & \\
\hline \multicolumn{6}{|l|}{ Location } \\
\hline Cardia & $495 / 141$ & $79 / 40$ & 1.00 & $1.84(1.20-2.83)$ & $<0.01$ \\
\hline Non-cardia & $495 / 292$ & $79 / 101$ & 1.00 & $2.18(1.57-3.04)$ & $<0.01$ \\
\hline \multicolumn{6}{|l|}{ Vascular invasion } \\
\hline Absent & $495 / 265$ & $79 / 98$ & 1.00 & $2.33(1.67-3.26)$ & $<0.01$ \\
\hline Present & $495 / 168$ & $79 / 43$ & 1.00 & $1.60(1.06-2.42)$ & 0.03 \\
\hline Lymph node metastasis & & & 1.00 & & \\
\hline Absent & $495 / 138$ & $79 / 35$ & 1.00 & $1.59(1.02-2.47)$ & 0.04 \\
\hline Present & $495 / 295$ & $79 / 106$ & 1.00 & $2.25(1.63-3.12)$ & $<0.01$ \\
\hline Liver metastasis & & & 1.00 & & \\
\hline Absent & $495 / 391$ & $79 / 121$ & 1.00 & $1.94(1.42-2.65)$ & $<0.01$ \\
\hline Present & $495 / 42$ & $79 / 20$ & 1.00 & $3.34(1.84-6.08)$ & $<0.01$ \\
\hline \multicolumn{6}{|l|}{ Peritoneal dissemination } \\
\hline Absent & $495 / 380$ & $79 / 123$ & 1.00 & $2.04(1.49-2.79)$ & $<0.01$ \\
\hline Present & $495 / 53$ & $79 / 18$ & 1.00 & $2.13(1.18-3.84)$ & 0.01 \\
\hline \multicolumn{6}{|l|}{ TNM stage } \\
\hline IA & $495 / 75$ & $79 / 21$ & 1.00 & $1.75(1.01-3.00)$ & 0.04 \\
\hline IB & $495 / 60$ & $79 / 19$ & 1.00 & $2.01(1.13-3.57)$ & 0.01 \\
\hline$\|$ & $495 / 83$ & $79 / 24$ & 1.00 & $1.78(1.06-2.98)$ & 0.03 \\
\hline III & $495 / 127$ & $79 / 46$ & 1.00 & $2.30(1.52-3.49)$ & $<0.01$ \\
\hline IV & $495 / 88$ & $79 / 31$ & 1.00 & $2.29(1.42-3.69)$ & $<0.01$ \\
\hline
\end{tabular}

${ }^{\dagger}$ Adjusted for age, sex, and $H$. pylori infection.

Abbreviations: $O R$, odds ratio; $C l$, confidence interval.

luciferase activities, compared with that of SNP309T allele in all three types of cell lines (all $P<0.01$ ). Furthermore, $H$. pylori LPS $1 \mu \mathrm{g} / \mathrm{ml}$ significantly increased the relative luciferase activity, compared with untreated controls in AGS cell, no matter whether SNP309T or SNP309G allele was transfected $(P<0.01$, Figure 3$)$.

\section{Meta-analysis of association between MDM2 promoter SNP309 polymorphism and gastric carcinoma}

We identified 4 published studies of the MDM2 SNP309 and gastric carcinoma susceptibility. With the combined data from those previous studies and from our current study, this meta-analysis included 1935 cases and 2953 controls. The characteristics of these studies are summarized in Additional file 1: Table S1. The studies are all Asian populations. As shown in Additional file 2: Table S2 and Additional file 3: Table S3, compared with the SNP309T/T genotype, the SNP309G/G genotype was associated with a significant increased risk of gastric carcinoma (OR, 1.57; 95\% CI, 1.08-2.29; $P=0.02$ ), whereas the SNP309T/G genotype was not associated significant with the risk of gastric carcinoma (OR, 1.08; $95 \% \mathrm{CI}, 0.83-1.41 ; P=0.57)$. In the recessive model, the
SNP309G/G homozygote was associated with a 1.51 fold increased risk of gastric carcinoma, compared with the $\mathrm{T}$ carriers $(95 \% \mathrm{CI}, 1.21-1.89 ; P<0.01)$. However, in the dominant model, the $\mathrm{G}$ carriers was associated with a 1.28-fold increased risk of gastric carcinoma, compared with the SNP309T/T genotype (95\% CI, 0.88-1.86; $P=0.19$ ). All stratified analyses are based on the recessive model. When stratified analysis by location of gastric carcinoma, the SNP309G/G homozygote was associated with a increased risk of gastric carcinoma, both in cardiac (OR, 1.65; 95\% CI, 1.12-2.43; $P=0.01)$ and non- cardiac gastric carcinoma (OR, 1.78; 95\% CI, 1.42-2.23; $P<0.01$ ) compared with the T carriers. Stratified by the histologic subtype, the SNP309G/G homozygote was associated with a 1.37-fold significant increased risk of intestinal carcinoma (95\% CI, 1.04-1.81; $P=0.03$ ), but not the diffuse carcinoma (OR, 1.05; 95\% CI, 0.76-1.43; $P=0.78$ ).

The publication bias of included studies was assessed by the Egger's test, and the results did not show evidence of publication bias ( $\mathrm{t}, 1.42 ; P=0.25$ for $\mathrm{T} / \mathrm{G}$ vs $\mathrm{T} / \mathrm{T}, \mathrm{t}, 2.62 ; P=0.08$ for $\mathrm{G} / \mathrm{G}$ vs $\mathrm{T}$ carriers, and $\mathrm{t}, 2.08$; $P=0.13$ for $\mathrm{G}$ carriers vs TT) except for $\mathrm{G} / \mathrm{G}$ vs $\mathrm{T} / \mathrm{T}$ (t, 3.40; $P=0.04)$. 

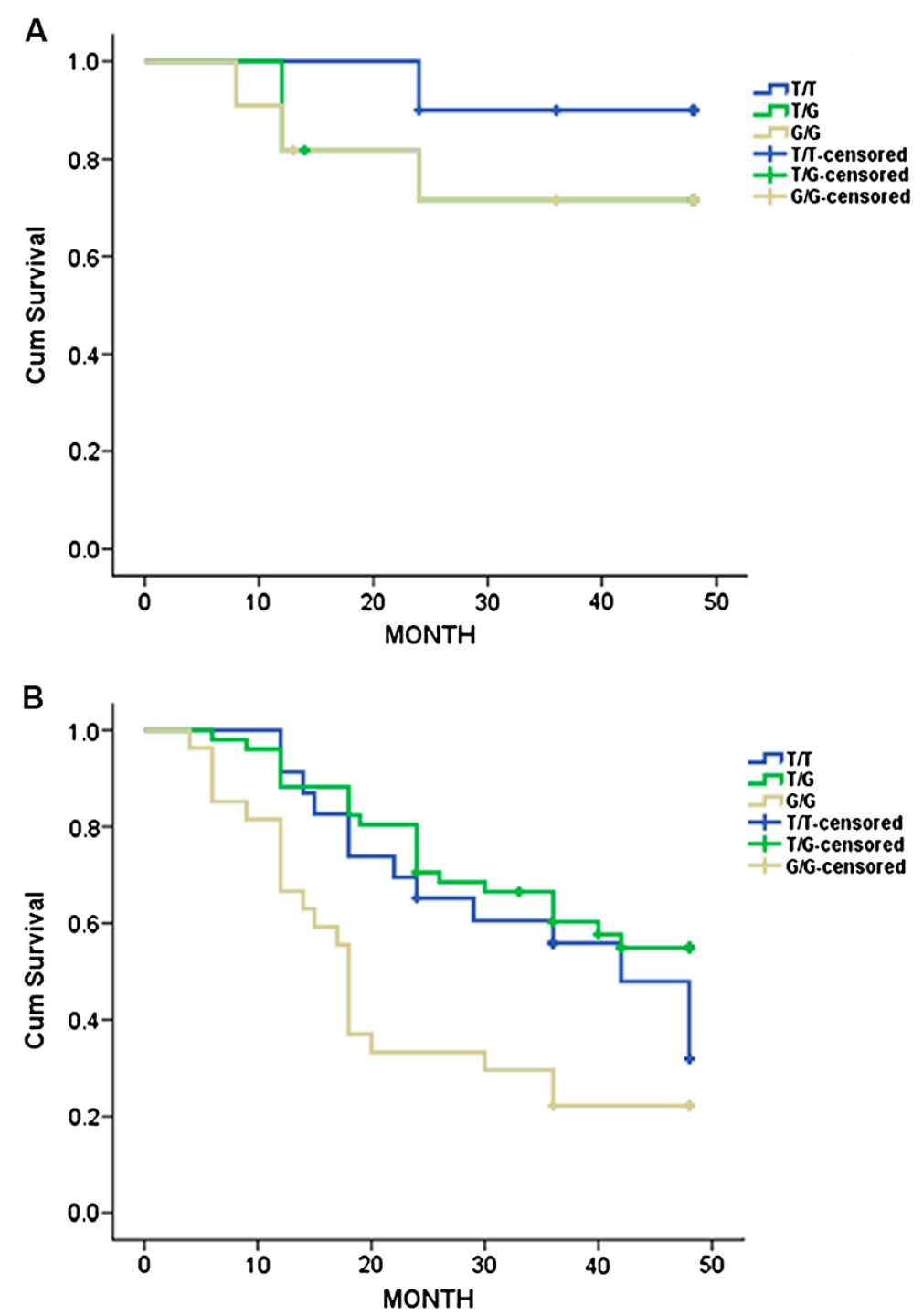

Figure 1 Association between MDM2 SNP309 and survival of patients with gastric carcinoma according to TNM classification. (A) Kaplan-Meier survival curves of 32 patients with TNM I stage according to MDM2 SNP309 genotypes $(P=0.50)$. (B) Kaplan-Meier survival curves of 101 patients with TNM II-IV stages according to MDM2 SNP309 genotypes $(P<0.01)$.

\section{Discussion}

It has been reported that the MDM2 SNP309G variant is bound more efficiently by Sp1 than $309 \mathrm{~T}$ allele, which increases MDM2 protein expression levels and weakens the p53 function [7]. So far, no consistent conclusion has been reached on the association between $M D M 2$ polymorphism and gastric carcinoma risk [19-23], and there has been only one study reporting that $M D M 2$ polymorphism is associated with both an increased susceptibility and poor prognosis of gastric carcinoma [19]. In our present large case-control analysis, we found that the SNP309G/G genotype was significant associated with gastric carcinoma risk and poor clinical prognosis. In addition, our metaanalysis also indicated that the MDM2 SNP309G/G genotype was associated significantly with an increased risk of gastric carcinoma, and provided further evidence indicating an association between this functional polymorphism and gastric carcinoma susceptibility. We also showed that the $\mathrm{T}$ to $\mathrm{G}$ substitution of this polymorphism significantly enhanced the transcription activity of the MDM2 gene in vitro.

It has been reported that MDM2 SNP309 is associated with poor prognosis of different kinds of carcinomas [32-34], and that SNPG/G is associated with shortened survival of patients with advanced gastric carcinoma [19]. In agreement with previous studies, we also found MDM2 SNP309G/G independently predicted poor prognosis of gastric carcinoma.

Intriguingly, we found that individuals with both MDM2 SNP309G/G and H. pylori infection conferred a 


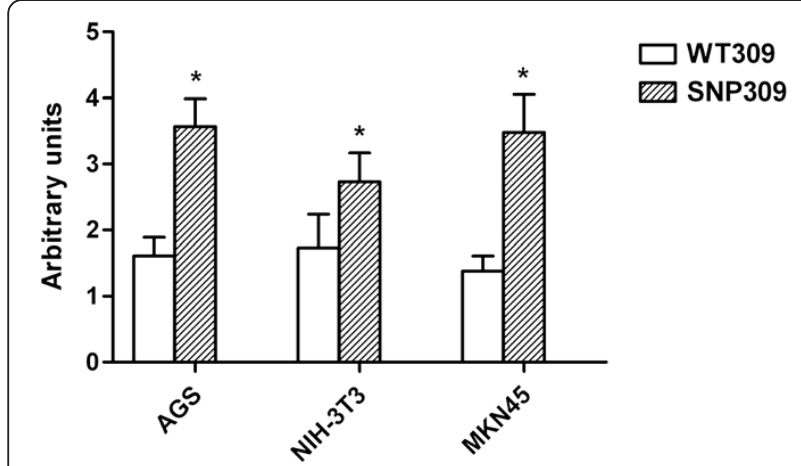

Figure 2 Effect of MDM2 SNP309 in the transcriptional activity of MDM2 promoter. Data are expressed as mean and standard deviation of three independent experiments. WT309, cells transfected with allele 309 T; SNP309, cells transfected with allele $309 \mathrm{G}$; * $P<0.01$, compared with WT309.

synergistic effect for developing gastric carcinoma with an OR of 2.44, suggesting a joint effect between $M D M 2$ polymorphism and $H$. pylori infection. $H$. pylori itself has been identified as the cause of gastric carcinoma and was classified as a Class I human carcinogen by the WHO [12,35-37]. It has reported that $H$. pylori infection increases the expression of MDM2 protein in the gastric mucosa [38,39], and the overexpression inhibits p53 function of tumor suppression [5]. It has been shown that $H$. pylori is able to phosphorylate and active MDM2 and subsequent degradation of p53 by activating AKT1 in gastric epithelial cells [40]. In addition, the cag PAI also contributes to p53 inactivation because individuals infected with $H$ pylori cagA strains have a higher likelihood of harboring p53 mutations [41]. Therefore, individuals with both $M D M 2$ SNP309G/G genotypes and $H$. pylori infection are expected to have a higher risk in development of gastric carcinoma.

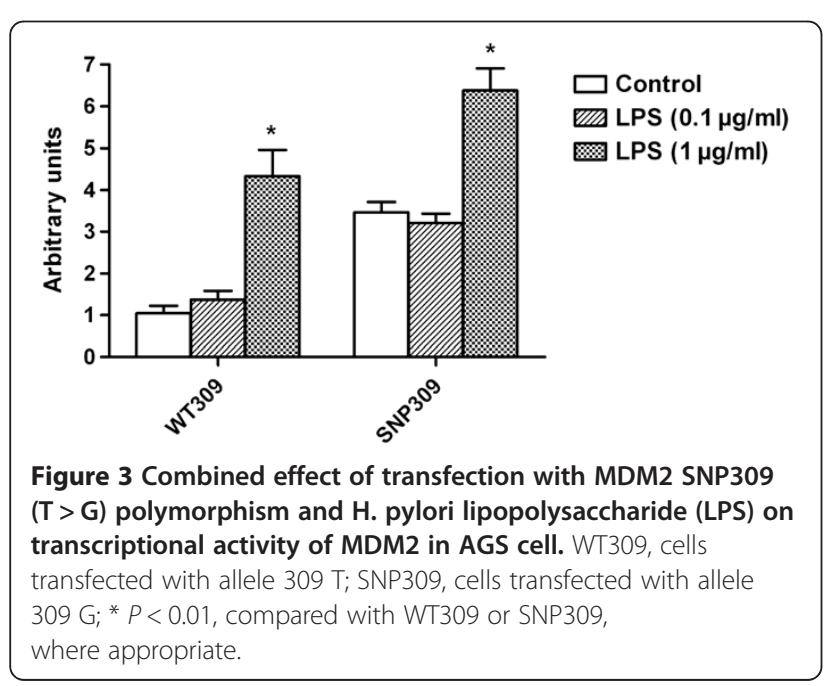

The association of $H$. pylori vacuolating cytotoxin A (VacA) or cytotoxin associated gene A protein (CagA) with gastric carcinoma has been extensively reported [42-44]. Recent studies have reported that LPS, another virulence factor of $H$. pylori, is closely related to gastric carcinoma [16,17]. Like E. coli LPS, H. pylori LPS is also composed of the specific polysaccharide, core polysaccharide and lipid A composition, and it has a certain toxicity and immune regulation, immune stimulation. Compared with E. coli LPS, H. pylori LPS has a lower endotoxin activity, and the ability of stimulating macrophages to produce pro-inflammatory factors and the role of nitric oxide are all weaker; however, it is the weak activity to endotoxin that sustains chronic gastric inflammation $[45,46]$. In the present study, we found that $H$. pylori LPS elevated the transcriptional activity of the gene MDM2, both in MDM2 WT309T and SNP309G. It has been reported that $H$. pylori LPS promotes colonization of $H$. pylori in the mucus layer adjacent to the gastric epithelial surface by interacting specifically with trefoil factor 1 in the stomach [15], and enhances proliferation and progression of gastric carcinoma by attenuating the antitumor activity and IFN- $\gamma$-mediated cellular immunity [16,17]. Our data suggested that the synergistic effect by MDM2 SNP309 (T > G) polymorphism and $H$. pylori infection may attribute to $H$. pylori LPS although further investigation is required.

\section{Conclusion}

In conclusion, the MDM2 SNP309 polymorphism is associated with gastric carcinoma risk and poor prognosis in Chinese patients. In addition, there is a joint effect of MDM2 SNP309G/G allele and H. pylori infection for the development of gastric carcinoma, which may attribute to H. pylori LPS.

\section{Additional files}

Additional file 1: Table S1. Characteristics of studies included in the current meta-analysis.

Additional file 2: Table S2. Result of meta-analysis of the association between MDM2 SNP309 polymorphism and gastric carcinoma risk.

Additional file 3: Table S3. Stratified of meta-analysis of the association between MDM2 SNP309 polymorphism and gastric carcinoma risk.

\section{Competing interests}

The authors declare that they have no competing interests.

Authors' contribution

GXZ XYW and RHS conceived and designed the experiments. XLP YQL JF and $\mathrm{BH}$ performed the experiments. XLP YQL and JF analyzed the data. GXZ contributed reagents/materials/analysis tools. XLP and YQL wrote the paper. All authors read and approved the final manuscript. 


\section{Acknowledgments}

This study was supported by Natural Science Funds of China (No. 81072032), Natural Science Funds of Jiangsu Province, China (No. BK2010194), and Postgraduates Research and Innovation Program of Jiangsu Province, P. R China (No. CXZZ11_0710).

\section{Author details}

1Department of Gastroenterology, the First Affiliated Hospital of Nanjing Medical University, Nanjing 210029, China. ${ }^{2}$ Department of General Surgery, the First Affiliated Hospital of Nanjing Medical University, Nanjing, China. ${ }^{3}$ Department of Gastroenterology, Changzhou No.2 People's Hospital Affiliated with Nanjing Medical University, Changzhou 213000, China.

Received: 17 November 2012 Accepted: 4 March 2013

Published: 18 March 2013

\section{References}

1. Jin S, Levine AJ: The p53 functional circuit. J Cell Sci 2001, 114:4139-4140

2. Soussi T, Lozano G: p53 mutation heterogeneity in cancer. Biochem Biophys Res Commun 2005, 331:834-842

3. Iwakuma T, Lozano G: MDM2, an introduction. Mol Cancer Res 2003 1:993-1000.

4. Lozano G: Mouse models of p53 functions. Cold Spring Harb Perspect Biol 2010, 2:a001115

5. Chen J, Wu X, Lin J, Levine AJ: mdm-2 inhibits the G1 arrest and apoptosis functions of the p53 tumor suppressor protein. Mol Cell Biol 1996, 16:2445-2452

6. Haupt $Y$, Barak Y, Oren M: Cell type-specific inhibition of p53-mediated apoptosis by MDM2. EMBO J 1996, 15:1596-1606.

7. Bond GL, Hu W, Bond EE, Robins H, Lutzker SG, Arva NC, Bargonetti J, Bartel F, Taubert H, Wuerl P, Onel K, Yip L, Hwang SJ, Strong LC, Lozano G, Levine $\mathrm{AJ}$ : A single nucleotide polymorphism in the MDM2 promoter attenuates the p53 tumor suppressor pathway and accelerates tumor formation in humans. Cell 2004, 119:591-602.

8. Bond GL, Hirshfield KM, Kirchhoff T, Alexe G, Bond EE, Robins H, Bartel F, Taubert H, Wuerl P, Hait W, Toppmeyer D, Offit K, Levine AJ: MDM2 SNP309 accelerates tumor formation in a gender-specific and hormonedependent manner. Cancer Res 2006, 66:5104-5110.

9. Dharel N, Kato N, Muroyama R, Moriyama M, Shao RX, Kawabe T, Omata M: MDM2 promoter SNP309 is associated with the risk of hepatocellular carcinoma in patients with chronic hepatitis C. Clin Cancer Res 2006, 12:4867-4871.

10. Grochola LF, Müller TH, Bond GL, Taubert H, Udelnow A, Würl P: MDM2 SNP309 associates with accelerated pancreatic adenocarcinoma formation. Pancreas 2010, 39:76-80.

11. Yarden RI, Friedman E, Metsuyanim S, Olender T, Ben-Asher E, Papa MZ: MDM2 SNP309 accelerates breast and ovarian carcinogenesis in BRCA1 and BRCA2 carriers of Jewish-Ashkenazi descent. Breast Cancer Res Treat 2008, 111:497-504.

12. Parsonnet J, Friedman GD, Vandersteen DP, Chang Y, Vogelman JH, Orentreich N, Sibley RK: Helicobacter pylori infection and the risk of gastric carcinoma. N Engl J Med 1991, 325:1127-1131.

13. Haruma K, Komoto K, Kamada T, Ito M, Kitadai Y, Yoshihara M, Sumii K, Kajiyama G: Helicobacter pylori infection is a major risk factor for gastric carcinoma in young patients. Scand J Gastroenterol 2000, 35:255-259.

14. Cover TL, Blaser MJ: Helicobacter pylori in health and disease. Gastroenterology 2009, 136:1863-1873.

15. Reeves EP, Ali T, Leonard P, Hearty S, O'Kennedy R, May FE, Westley BR, Josenhans C, Rust M, Suerbaum S, Smith A, Drumm B, Clyne M: Helicobacter pylori lipopolysaccharide interacts with TFF1 in a pH-dependent manner. Gastroenterology 2008, 135:2043-2054. 2054. e1-2.

16. Chochi K, Ichikura T, Kinosthita M, Majima T, Shinomiya N, Tsujimoto H, Kawabata T, Sugasawa H, Ono S, Seki S, Mochizuki H: Helicobacter pylori augments growth of gastric cancers via the lipopolysaccharide-toll-like receptor 4 pathway whereas its lipopolysaccharide attenuates antitumor activities of human mononuclear cells. Clin Cancer Res 2008, 14:2909-2917

17. Yokota S, Okabayashi T, Rehli M, Fujii N, Amano K: Helicobacter pylori lipopolysaccharides upregulate toll-like receptor 4 expression and proliferation of gastric epithelial cells via the MEK1/2-ERK1/2 mitogenactivated protein kinase pathway. Infect Immun 2010, 78:468-476.
18. Peek RM Jr, Crabtree JE: Helicobacter infection and gastric neoplasia. J Pathol 2006, 208:233-248.

19. Ohmiya N, Taguchi A, Mabuchi N, Itoh A, Hirooka Y, Niwa Y, Goto H: MDM2 promoter polymorphism is associated with both an increased susceptibility to gastric carcinoma and poor prognosis. J Clin Oncol 2006, 24:4434-4440.

20. Yang M, Guo Y, Zhang X, Miao X, Tan W, Sun T, Zhao D, Yu D, Liu J, Lin D: Interaction of P53 Arg72Pro and MDM2 T309G polymorphisms and their associations with risk of gastric cardia cancer. Carcinogenesis 2007 28:1996-2001.

21. Cao YY, Zhang XF, Guo W, Wang R, Ge H, Zhang JH: Association of the MDM2 polymorphisms with susceptibility of esophageal squamous cell carcinoma and that of gastric cardiac adenocarcinoma. Tumor 2007, 8:628-632.

22. Cho YG, Choi BJ, Song JH, Kim CJ, Cao Z, Nam SW, Lee JY, Park WS: No association of MDM2 T309G polymorphism with susceptibility to Korean gastric cancer patients. Neoplasma 2008, 55:256-260.

23. Wang $X$, Yang J, Ho B, Yang Y, Huang Z, Zhang Z, Zhang G: Interaction of Helicobacter pylori with genetic variants in the MDM2 promoter is associated with gastric cancer susceptibility in Chinese patients. Helicobacter 2009, 14:114-119.

24. Edge SB, Byrd DR, Compton CC, Fritz AG, Greene FL, Trotti A: eds: American Joint Committee on Cancer Staging Manual. 7th edition. New York: Springer: 2009 .

25. Lauren P: The two histological main types of gastric carcinoma: diffuse and so-called intestinal-type carcinoma. Acta Pathol Microbiol Scand 1965, 64:31-49.

26. Wang $X Y$, Yang $Y$, Shi $R H$, Ho B, Wang HD, Zhang GX: An evaluation of a serologic test with a current infection marker of Helicobacter pylori before and after eradication therapy in Chinese. Helicobacter 2008, 13:49-55

27. Prendergast MM, Kosunen TU, Moran AP: Development of an immunoassay for rapid detection of ganglioside GM(1) mimicry in Campylobacter jejuni strains. J Clin Microbiol 2001, 39:1494-1500.

28. Lee $\mathrm{CH}$, Tsai CM: Quantification of bacterial lipopolysaccharides by the purpald assay: measuring formaldehyde generated from 2-keto-3deoxyoctonate and heptose at the inner core by periodate oxidation. Anal Biochem 1999, 267:161-168.

29. Mantel N, Haenszel W: Statistical aspects of the analysis of data from retrospective studies of disease. J Natl Cancer Inst 1959, 22:719-748.

30. DerSimonian R, Laird N: Meta-analysis in clinical trials. Control Clin Trials 1986, 7:177-188

31. Egger M, Davey Smith $G$, Schneider M, Minder C: Bias in meta-analysis detected by a simple, graphical test. BMJ 1997, 315:629-634

32. Schmidt MK, Tommiska J, Broeks A, van Leeuwen FE, Van't Veer LJ, Pharoah $P D$, Easton DF, Shah M, Humphreys M, Dörk T, Reincke SA, Fagerholm R, Blomqvist C, Nevanlinna H: Combined effects of single nucleotide polymorphisms TP53 R72P and MDM2 SNP309, and p53 expression on survival of breast cancer patients. Breast Cancer Res 2009, 11:R89.

33. Han JY, Lee GK, Jang DH, Lee SY, Lee JS: Association of p53 codon 72 polymorphism and MDM2 SNP309 with clinical outcome of advanced nonsmall cell lung cancer. Cancer 2008, 113:799-807.

34. Gryshchenko I, Hofbauer S, Stoecher M, Daniel PT, Steurer M, Gaiger A Eigenberger K, Greil R, Tinhofer I: MDM2 SNP309 is associated with poor outcome in B-cell chronic lymphocytic leukemia. J Clin Oncol 2008, 26:2252-2257

35. Shikata K, Doi Y, Yonemoto K, Arima H, Ninomiya T, Kubo M, Tanizaki Y, Matsumoto T, lida M, Kiyohara Y: Population-based prospective study of the combined influence of cigarette smoking and Helicobacter pylori infection on gastric cancer incidence: the Hisayama Study. Am J Epidemiol 2008, 168:1409-1415

36. Huang JQ, Sridhar S, Chen Y, Hunt RH: Meta-analysis of the relationship between Helicobacter pylori seropositivity and gastric cancer. Gastroenterology 1998, 114:1169-1179.

37. Blaser MJ, Parsonnet J: Parasitism by the "slow" bacterium Helicobacter pylori leads to altered gastric homeostasis and neoplasia. J Clin Invest 1994, 94:4-8.

38. Kodama M, Fujioka T, Murakami K, Okimoto T, Sato R, Watanabe K, Nasu M: Eradication of Helicobacter pylori reduced the immunohistochemical detection of p53 and MDM2 in gastric mucosa. $J$ Gastroenterol Hepatol 2005, 20:941-946. 
39. Nakajima N, Ito Y, Yokoyama K, Uno A, Kinukawa N, Nemoto N, Moriyama M: The Expression of Murine Double Minute 2 (MDM2) on Helicobacter pylori-Infected Intestinal Metaplasia and Gastric Cancer. J Clin Biochem Nutr 2009, 44:196-202

40. Wei J, Nagy TA, Vilgelm A, Zaika E, Ogden SR, Romero-Gallo J, Piazuelo MB, Correa P, Washington MK, El-Rifai W, Peek RM, Zaika A: Regulation of p53 tumor suppressor by Helicobacter pylori in gastric epithelial cells. Gastroenterology 2010, 139:1333-1343.

41. Shibata A, Parsonnet J, Longacre TA, Garcia MI, Puligandla B, Davis RE, Vogelman JH, Orentreich N, Habel LA: CagA status of Helicobacter pylori infection and p53 gene mutations in gastric adenocarcinoma. Carcinogenesis 2002, 23:419-424.

42. Huang JQ, Zheng GF, Sumanac K, Irvine EJ, Hunt RH: Meta-analysis of the relationship between cagA seropositivity and gastric cancer. Gastroenterology 2003, 125:1636-1644.

43. Parsonnet J, Friedman GD, Orentreich N, Vogelman H: Risk for gastric cancer in people with CagA positive or CagA negative Helicobacter pylori infection. Gut 1997, 40:297-301.

44. Wen S, Moss SF: Helicobacter pylori virulence factors in gastric carcinogenesis. Cancer Lett 2009, 282:1-8.

45. Ogawa T, Asai Y, Sakai Y, Oikawa M, Fukase K, Suda Y, Kusumoto S, Tamura T: Endotoxic and immunobiological activities of a chemically synthesized lipid A of Helicobacter pylori strain 206-1. FEMS Immunol Med Microbiol 2003, 36:1-7.

46. Matsuyama N, Kirikae T, Kirikae F, Hashimoto M, Amanot K, Hayashi S, Hirai Y, Kubota T, Nakano M: Non-standard biological activities of lipopolysaccharide from Helicobacter pylori. J Med Microbiol 2001, 50:865-869.

doi:10.1186/1471-2407-13-126

Cite this article as: Pan et al: A functional polymorphism T309G in MDM2 gene promoter, intensified by Helicobacter pylori lipopolysaccharide, is associated with both an increased susceptibility and poor prognosis of gastric carcinoma in Chinese patients. BMC Cancer 2013 13:126.

\section{Submit your next manuscript to BioMed Central and take full advantage of:}

- Convenient online submission

- Thorough peer review

- No space constraints or color figure charges

- Immediate publication on acceptance

- Inclusion in PubMed, CAS, Scopus and Google Scholar

- Research which is freely available for redistribution 